\title{
Neuropatia óptica isquêmica posterior como complicação pós-cirurgia de blefaroplastia
}

\author{
Posterior ischemic optic neuropathy as a \\ complication after blepharoplasty surgery
}

Maria Helena Lopes Amigo ${ }^{1}$, Wagner Ghirelli²

\section{$\underline{\text { ReSUMO }}$}

Descrevemos um caso de perda visual unilateral em paciente de 80 anos após cirurgia de blefaroplastia bilateral superior e inferior com o olho direito apresentando intensa hemorragia pré-orbitária. O exame oftalmológico apresentou quadro clínico de neuropatia óptica isquêmica posterior com hemianopsia superior e ressonância magnética de órbita normal. Comentamos alguns possíveis mecanismos e análise da evolução da camada de fibras nervosas retinianas pela tomografia de coerência óptica.

Descritores: Cegueira; Blefaroplastia/efeitos adversos; Testes de campo visual/métodos; Neuropatia óptica isquêmica; Tomografia de coerência óptica; Relato de caso

\section{ABstraCt}

A case of unilateral visual loss and right eye presenting intense pre-orbital bleeding was described in patient 80 years old, after upper and lower bilateral blepharoplasty surgery. Ophthalmologic examination showed clinical profile of posterior ischemic optic neuropath with superior hemianopsia and magnetic resonance imaging (MRI) of normal orbit. Comments are made on possible mechanism involved in the process as well as analysis on the evolution of the retinal nerve layer by optical coherence tomography.

Keywords: Blindness; Blepharoplasty/adverse effects;Visual field tests/methods; Optic neuropathy, ischemic; Tomography, optical coherence; Case report

\footnotetext{
'Médica residente do primeiro ano de oftalmologia do Centro de Oftalmologia Tadeu Cvintal - São Paulo (SP), Brasil; ${ }^{2}$ Médico chefe do departamento de retina e vítreo do Centro de Oftalmologia Tadeu Cvintal - São Paulo (SP), Brasil.

Centro de Oftalmologia Tadeu Cvintal - São Paulo (SP), Brasil

Os autores declaram inexistir conflitos de interesse
}

Recebido para publicação em: 5/11/2010 - Aceito para publicação em 4/7/2011 


\section{INTRODUÇÃO}

A cirurgia de blefaroplastia é uma cirurgia muito realizada na plástica oftalmológica, sendo que uma das complicações mais graves relacionadas ao pós-operatório corresponde à perda parcial ou total da visão. Demere em 1974 publicou pela primeira vez uma estatística baseada em pesquisa realizada nos Estados Unidos, que mostrou uma incidência de $0,04 \%$ de perda súbita de visão após cirurgia de blefaroplastia ${ }^{(1)}$. Em 1998, 1200 cirurgiões plásticos filiados a Sociedade Brasileira de Cirurgia Plástica responderam um questionário sobre perda da visão após blefaroplastia, oito casos foram reportados, sendo que em nenhum deles a neurite óptica isquêmica posterior foi citada ${ }^{(2)}$. Na literatura nacional brasileira sobre cirurgia plástica oftalmológica é citado à ocorrência de hematoma intraorbitário como causa de perda da função visual ${ }^{(3)}$.

Descreveremos a seguir um relato de caso sobre a perda visual unilateral pós-blefaroplastia causada por neurite óptica isquêmica posterior e seus possíveis mecanismos.

\section{Relato de caso}

Paciente, feminino, 80 anos, compareceu em nosso serviço dois dias após realização de blefaroplastia superior e inferior bilateral, apresentando intensa hemorragia pré-orbitária do lado direito e intensa dificuldade de abertura dos olhos. A paciente relatava ter sido operada duas vezes no mesmo dia do olho direito (OD) devido à hematoma intenso na pálpebra inferior. Nos antecedentes sistêmicos pessoais não apresentava: hipertensão arterial sistêmica (HAS), diabetes mellitus e coagulopatia. Nos antecedentes oftalmológicos relatava episódio prévio de trombose venosa em olho esquerdo (OE).

Ao exame oftalmológico apresentava acuidade visual (AV) em (OD) LogMAR 1.30 e (OE) LogMAR 0.40 . Ao exame externo apresentava intensa quemose de pálpebra inferior e bulbo ocular com motilidade extrínseca normal e defeito pupilar aferente relativo +1 em OD. Não havia proptose. A biomicroscopia apresentava córnea clara, catarata nuclear $+1 / 4$ em OD e pseudofácica OE. A tonometria de aplanação no OD era de $14 \mathrm{mmHg}$ e OE $20 \mathrm{mmHg}$. A fundoscopia em OD: sem alterações de nervo óptico e mácula, e OE: presença de vasos colaterais na papila (sequela de trombose da veia central da retina).

Foram solicitados os seguintes exames: campimetria visual computadorizada, angiofluores-

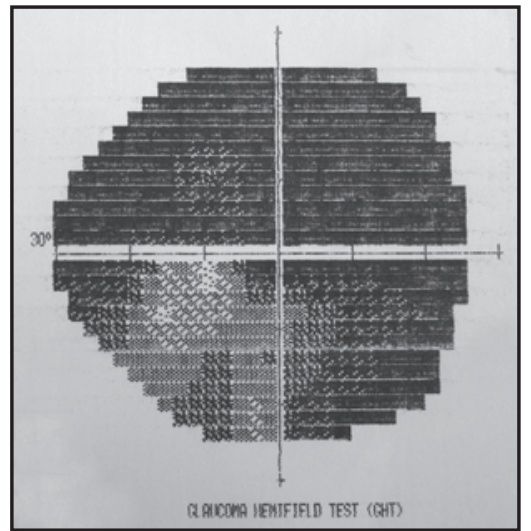

Figura 1: Campimetria computadorizada olho direito mostrando perda difusa da sensibilidade central com preservação relativa do setor nasal inferior

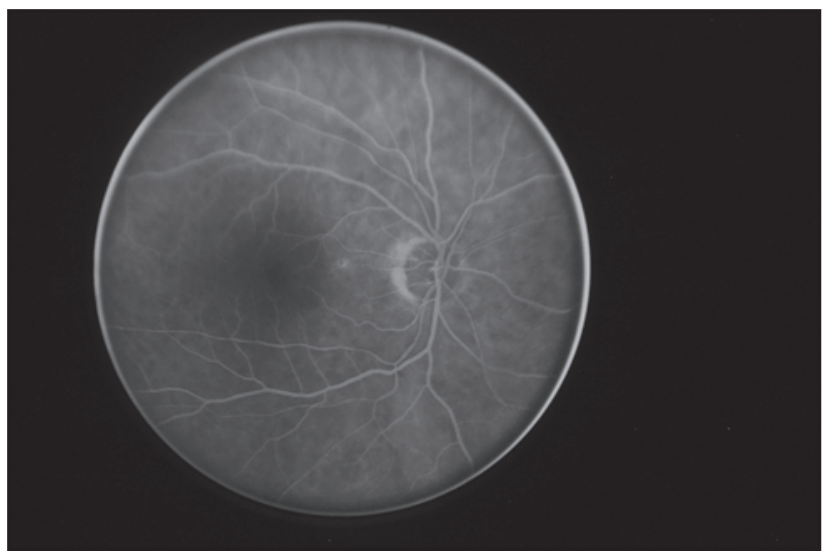

Figura 2: Angiofluoresceinografia retiniana normal

ceinografia, tomografia de coerência óptica (TCO) com análise de camada de fibras nervosas e ressonância magnética de órbita. A campimetria visual apresentou em OD: perda difusa da sensibilidade central com preservação relativa do setor nasal inferior (Figura 1), e em OE: Constrição do campo visual. A angiofluoresceinografia mostrou tempo circulatório normal e ausência de áreas de hipo ou hiperfluorescência anômalas e papila com fluorescência normal (Figura 2). A TCO de camadas de fibras nervosas dentro do padrão de normalidade na primeira visita e repetindo o exame após dois meses da cirurgia mostrou diminuição da camada de fibras nervosas nos quadrantes inferior e temporal (Figura 3). A ressonância magnética: bulbo ocular e estruturas intraorbitárias simétricas sem sinais de captação, nervo óptico e quiasma óptico sem alterações e parênquima encefálico sem evidências de anomalias (Figura 4). 

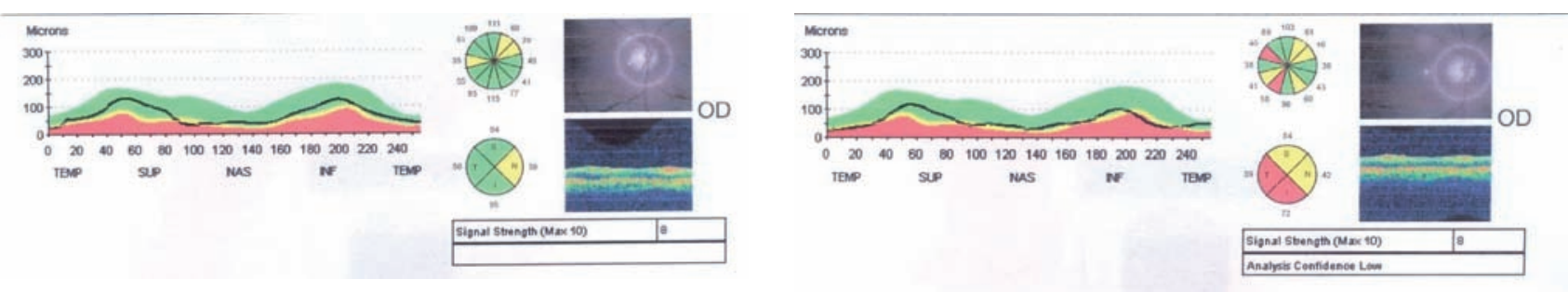

Figura 3: A) TCO na primeira semana pós-cirurgia mostrando espessura normal da camada de fibras nervosas; B) TCO no segundo mês com diminuição da camada de fibras nervosas nos quadrantes inferior e temporal (cor vermelha)

\section{Dıscussão}

A neuropatia optica isquêmica posterior (NOIP, é uma entidade rara cujo diagnóstico deve afastar outras causas de neuropatia óptica retrobulbar, tais como inflamatórias, tóxicas e compressivas. A NOIP foi classificada em três subtipos (arterítica, não arterítica e perioperatório) por Sadda et al. ${ }^{(4)}$, que revendo 72 pacientes com NOIP encontrou 28 póscirurgia sendo que $50 \%$ eram pós-cirurgia espinhal, e a outra metade era dividida em cirurgias cardíacas, cerebrovascular, ortopédicas, abdominal e oculares o que demonstra a baixa frequência pós-cirurgia pós-ocular ${ }^{(4)}$.

A paciente apresentou perda visual imediatamente após a cirurgia plástica no olho direito onde, segundo a paciente, sofreu duas intervenções no primeiro pósoperatório devido intenso edema na pálpebra inferior. O fato de no primeiro pós-operatório oftalmológico, que ocorreu no segundo pós-operatório, apresentar baixa visual com fundo de olho normal e defeito pupilar aferente caracterizou a neuropatia retrobulbar. O exame angioflurescêinográfico normal afastou a possibilidade de ter sido afetada a artéria central da retina como também a cabeça do nervo óptico. Os exames sanguíneos normais afastaram a causa inflamatória. A ressonância magnética de órbita que não mostrou hematomas, ou neoplasias afastou a causa compressiva. A campimetria computadorizada mostrando um padrão de perda difusa da sensibilidade central com preservação relativa do setor nasal inferior, vem complementar a suspeita de um processo vascular reforçando a hipótese diagnóstica de neuropatia óptica isquêmica posterior.

A etiologia da perda da visão pós-blefaroplastia pode estar relacionado à hemorragia intraorbitária, o tipo de anestesia e a técnica cirúrgica utilizada ${ }^{(2,-5)}$. Os casos citados na literatura são os seguintes: glaucoma, uveíte, úlcera de córnea, leucoma, tumor hipofisário, compressão intraoperatória do bulbo ocular, celulite orbitária e hematoma intranervo óptico ${ }^{(2)}$.

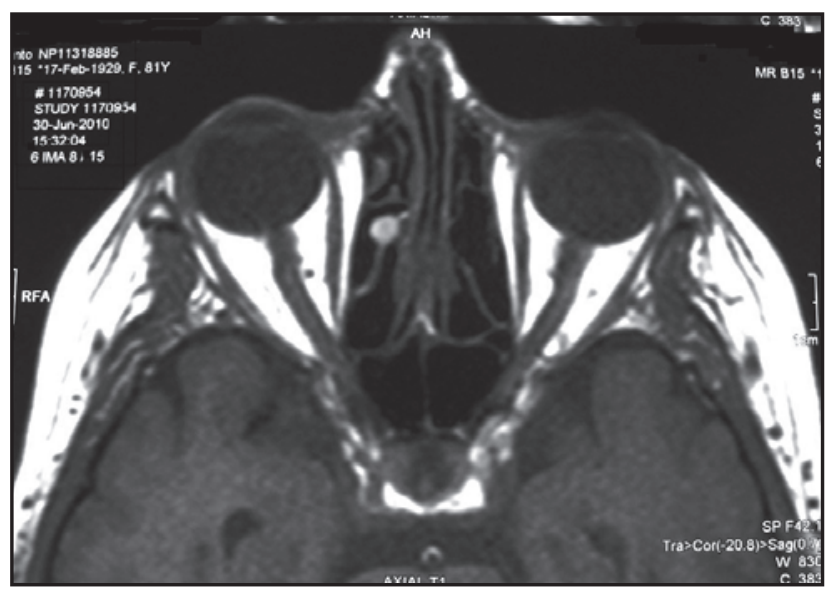

Figura 4: Ressonância magnética de órbita normal

O mecanismo mais relatado na literatura internacional foi a hemorragia retrobulbar com consequente comprometimento da circulação do nervo óptico e da retina pela artéria central da retina ${ }^{(2,5-8)}$. Outros mecanismos citados em menor frequência são glaucoma agudo e oclusão da veia central da retina $(\mathrm{OVCR})^{(5-8)}$. A paciente teve OVCR no olho contralateral mas não póscirurgia. A complicação de blefaroplastia que causa perda visual com fundo de olho normal, defeito pupilar aferente e perda difusa da sensibilidade central com preservação relativa do setor nasal inferior é a $\operatorname{NOIP}^{(9-14)}$.

A NOIP pós-blefaroplastia está associada a alterações orbitárias como hemorragias ${ }^{(12-14)}$, no caso a ressonância magnética era normal, levamos então a considerar o uso do anestésico local com vaso constritor, o qual promove a isquemia por comprometer as artérias principais responsáveis pela irrigação da parte orbitária do nervo óptico. Em contato com o cirurgião plástico fomos informados da não utilização de vasoconstritor na anestesia local e que a reoperação foi realizada para remoção de coágulo da pálpebra inferior sem acesso a cavidade orbitária. A partir desse fato achamos que apesar de não haver hematoma intraorbitário a deformação anatômica provocada pela hemorragia externa pode ter aumentado transitoria- 
mente a pressão intraorbitária causando isquemia.

$\mathrm{Na}$ literatura pesquisada encontramos um caso semelhante ao nosso publicado por Kordic $\mathrm{H}$ et al. em $2005^{(10)}$, onde o fator causal não foi encontrado. O que nos chamou atenção no artigo foi o padrão da campimetria visual muito semelhante ao nosso. A análise da progressão de perdas de fibras nervosas pelo TCO comprova o afinamento da camada inferior após dois meses do ocorrido o que talvez seja um padrão de lesão nesses pacientes.

Concluímos que um cuidado maior deva ser tomado com a hemostasia perioperatória, evitar utilização de anestesia com vasoconstritor em blefaroplastia principalmente inferior, cuidados especiais em pacientes idosos mesmo sem antecedentes de doenças vasculares sistêmicas.

\section{ReferêNCIAS}

1. DeMere M, Wood T, Austin W. Eye complications with blepharoplasty or other eyelid surgery. Plast Reconstr Surg. 1974;53(6):634-7.

2. Peruzzo M, Mélega JM. Cegueira pós-blefaroplastia estética. Rev Soc Bras Cir Plást (1986). 1988;3(2):138-43.

3. Moura EM. Blefaroplastia. In: Matayoshi S, Forno EA, Moura EM. Manual de cirurgia plástica ocular. São Paulo: Roca; 2004.

4. Sadda SR, Nee M, Miller N, Biousse V, Newman NJ, Kouzis A. Clinical spectrum of posterior ischemic optic neuropathy. Am J Ophthalmol. 2001;132(5):743-50.
5. Hartley J, Lester J, Schatten W. Acute retrobulbar hemorrhage during elective blepharoplasty. Its pathophysiology and management. Plast Reconstr Surg. 1973;52(1):8-15.

6. Heinze JB, Hueston JT. Blindness after blepharoplasty: mechanism and early reversal. Plast Reconstr Surg. 1978;61(3):347-54.

7. Hueston JT, Heinze JB.Successful early relief of blindness occurring after blefaroplasty. Case report. Plast Reconstr Surg. 1974;53(5):588-92.

8. Waller RR. Is blindness a realistic complication in blepharoplasty procedures? Ophthalmology. 1978;85(7 Pt 1):730-5.

9. Hayreh SS. Ischaemic optic neuropathy. Indian J Ophthalmol. 2000;48(3):171-94.

10. Kordic H, Flammer J, Mironow A, Killer HE. Perioperative posterior ischemic optic neuropathy as a rare complication of blepharoplasty. Ophthalmologica. 2005;219(3):185-8.

11. Killer HE, Forrer A, Blumer BK. Nonarteritic posterior ischemic optic neuropathy treated with optic sheath decompression. Report of two cases. Neuroophthalmology. 1998;19(2):101-6.

12. Mahaffey PJ, Wallace AF. Blindness following cosmetic blepharoplasty--a review. Br J Plast Surg. 1986;39(2):213-21.

13. Anderson RL. Bilateral visual after blepharoplasty. Arch Ophthalmol. 1981;99(12):2205.

14. Good CD, Cassidy LM, Moseley IF, Sanders MD. Posterior optic nerve infarction after lower lid blepharoplasty. J Neuroophthalmol. 1999;19(3):176-9.

\author{
Autor correspondente: \\ Wagner Ghirelli \\ Rua Maria Figueiredo, $\mathbf{n}^{\circ} 283$ \\ Tel:(21)3371-3324 \\ E-mail: wagnerghirelli@yahoo.com.br
}

